VOL. 63 (2001) [219-228]

\title{
PARABOLIC EQUATIONS WITH DISCONTINUOUS NONLINEARITIES
}

\author{
Giuseppina BARLetTa
}

\begin{abstract}
In this paper we deal with the homogeneous Cauchy-Dirichlet problem for a class of parabolic equations with either Carathéodory or discontinuous nonlinear terms. We then present an application and explicitly point out an existence result for a differential inclusion, which can be applied to the classical Stefan problem.
\end{abstract}

\section{INTRODUCTION}

Let $\Omega$ be an open, bounded, and connected subset of $\mathbf{R}^{n}$ having the cone property, let $T>0$, and let $Q_{T}=\Omega \times(0, T)$. Denote by $\mathcal{L}$ a linear, second order, uniformly parabolic operator and by $f$ a real valued function defined on $Q_{T} \times \mathbf{R}$.

The aim of this paper is to study the following Cauchy-Dirichlet problem:

$$
\begin{cases}\mathcal{L} u=f(x, t, u) & \text { in } Q_{T} \\ u(x, 0)=0 & \text { on } \Omega \\ u(x, t)=0 & \text { on } \partial \Omega \times(0, T)\end{cases}
$$

Adapting the approach previously used in $[14,15]$ to get existence results for elliptic equations with possibly discontinuous nonlinear terms, we establish here two existence theorems for the above problem and an existence theorem for a differential inclusion. These results are presented in Section 3, whereas Section 2 is devoted to basic definitions and preliminary results.

The first theorem deals with the case when the right-hand side $f$ is a Caratheodory function satisfying a suitable growth condition and is obtained through a fixed point result of Arino, Gautier, and Penot [2, Theorem 1]. The other treats a different case, where $f$ has a set of discontinuity points (with respect to $u$ ) which is allowed to be more than countable, but of Lebesgue measure zero.

As far as we know, previous existence theorems for parabolic equations and for parabolic

\section{Received 9th May, 2000}

The author wishes to thank Professor S.A. Marano for introducing her to the topics treated in this paper and for many stimulating conversations.

Copyright Clearance Centre, Inc. Serial-fee code: 0004-9727/01 \$A2.00+0.00. 
inclusions with discontinuous nonlinearities were obtained by using results on pseudomonotone operators and under the common assumption of the existence of an upper solution and a lower solution to the problem; see $[4,5,16]$ and the recent monograph [11]. Furthermore, the discontinuous term either is of the form $f+h$, where $f \in L_{\text {loc }}^{\infty}(\mathbf{R})$ and satisfies a $L^{q}\left(Q_{T}\right)$ boundedness condition with respect to a specified interval, while $h \in L^{2}\left(Q_{T}\right)$, (see [16]), or it is a function defined on $Q_{T} \times \mathbf{R} \times \mathbf{R} \times \mathbf{R}^{n}$ that satisfies some boundedness, continuity, and nondecreasing conditions (see [5]). In [9] an existence result is obtained by using variational methods. We adopt here a different approach, which basically follows the one previously employed in [14] to treat the elliptic case. Namely, we first pass to a multivalued problem and we look for a solution of it. The assumptions made then guarantee that such a function is also a weak solution of the original problem.

\section{Preliminaries}

Let $\Omega$ be an open, bounded, and connected subset of $\mathbf{R}^{n}, n \geqslant 3$, with the cone property, let $T>0$, and let $Q_{T}=\Omega \times(0, T)$. Given $p \in(1,+\infty)$ and $m \in N_{0}$ we denote by $W^{m, p}\left(Q_{T}\right)$ the space of functions $u \in L^{p}\left(Q_{T}\right)$ having derivatives (in the distributional sense) of the form $\partial_{t}^{r} \partial_{x}^{s} u$ with $r \in \mathbf{N}_{0}, s=\left(s_{1}, \ldots, s_{n}\right)$, and $|r+s|:=r+s_{1}+\cdots+s_{n} \leqslant m$, while $W_{p}^{2,1}\left(Q_{T}\right)$ stands for the space of functions $u \in L^{p}\left(Q_{T}\right)$ having derivatives (in the distributional sense) of the form $\partial_{t}^{r} \partial_{x}^{s} u$ where $2 r+s \leqslant 2$. On $W^{m, p}\left(Q_{T}\right)$ we consider the norm

$$
\|u\|_{m, p}=\sum_{|r+s| \leqslant m}\left\|\partial_{t}^{r} \partial_{x}^{s} u\right\|_{p}
$$

while $W_{p}^{2,1}\left(Q_{T}\right)$ is equipped with the norm

$$
\|u\|_{2,1, p}=\sum_{|\alpha| \leqslant 2}\left\|\partial_{x}^{\alpha} u\right\|_{p}+\left\|\partial_{t} u\right\|_{p}
$$

where $\alpha=\left(\alpha_{1} \ldots, \alpha_{n}\right)$ and

$$
\partial_{x}^{\alpha} u=\frac{\partial^{|\alpha|} u}{\partial_{x_{1}}^{\alpha_{1}} \cdots \partial_{x_{n}}^{\alpha_{n}}}, \partial_{t} u=\frac{\partial u}{\partial t}
$$

Finally, $W\left(Q_{T}\right)$ denotes the Banach space of functions $u \in W_{p}^{2,1}\left(Q_{T}\right)$ such that $u(x, 0)=$ 0 for every $x \in \Omega$ and $u(x, t)=0$ for every $(x, t) \in \partial \Omega \times(0, T)$. One clearly has $\|u\|_{1, p} \leqslant\|u\|_{2,1, p}$ whenever $u \in W_{p}^{2,1}\left(Q_{T}\right)$. The Rellich-Kondrachov theorem [1, p. 144] guarantees that the embedding

$$
W^{1, p}\left(Q_{T}\right) \longrightarrow L^{p^{*}}\left(Q_{T}\right)
$$


with $1 \leqslant p^{*}<(n+1) p /(n+1-p)$ if $p<n+1$ and $1 \leqslant p^{*}<\infty$ if $p \geqslant n+1$, is compact. Write $c_{1}$ for the smallest constant such that

$$
\|u\|_{p^{*}} \leqslant c_{1}\|u\|_{2,1, p} \quad \forall u \in W_{p}^{2,1}\left(Q_{T}\right)
$$

Let $\mathcal{L}$ be the linear, second order, uniformly parabolic differential operator defined by

$$
\mathcal{L}(u)=\frac{\partial u}{\partial t}-\sum_{i, j=1}^{n} a_{i j}(x, t) \frac{\partial^{2} u}{\partial x_{i} \partial x_{j}}+\sum_{i=1}^{n} a_{i}(x, t) \frac{\partial u}{\partial x_{i}}+a(x, t) u
$$

where $a_{i j}(i, j=1, \ldots, n)$ are real-valued continuous bounded functions defined on $Q_{T}$, $a_{i} \in L^{r}\left(Q_{T}\right)$, with

$$
r= \begin{cases}\max \{p, n+2\} & \text { if } p \neq n+2 \\ n+2+\varepsilon & \text { if } p=n+2(\varepsilon>0)\end{cases}
$$

$a \in L^{s}\left(Q_{T}\right)$, with

$$
s= \begin{cases}\max \left\{p, \frac{n+2}{2}\right\} & \text { if } p \neq \frac{n+2}{2} \\ \frac{n+2}{2}+\varepsilon & \text { if } p=\frac{n+2}{2}\end{cases}
$$

and $\sum_{i, j=1}^{n} a_{i j}(x, t) \xi_{i} \xi_{j} \geqslant \lambda \sum_{i=1}^{n} \xi_{i}^{2}$ for some $\lambda>0$, for every $(x, t) \in Q_{T}$ and every $\xi_{1}, \xi_{2}, \ldots, \xi_{n} \in \mathbf{R} .[13$, Theorem 9.1, p. 341] guarantees that the operator

$$
\mathcal{L}: W\left(Q_{T}\right) \longrightarrow L^{p}\left(Q_{T}\right)
$$

is bijective and that its inverse

$$
\mathcal{L}^{-1}: L^{p}\left(Q_{T}\right) \longrightarrow W\left(Q_{T}\right)
$$

is continuous. Therefore,

$$
\left\|\mathcal{L}^{-1}(v)\right\|_{2,1, p} \leqslant\left\|\mathcal{L}^{-1}\right\|\|v\|_{p} \quad \forall v \in L^{p}\left(Q_{T}\right)
$$

We recall the following proposition, which is easily obtained by using [7, Lemma 1$]$ and [10, Lemma 7.7].

Proposition 2.1. Let $u \in W\left(Q_{T}\right)$ and let $E$ be a measurable subset of $\mathbf{R}$ such that $|E|=0$. Then $\mathcal{L}(u)(x, t)-a(x, t) u(x, t)=0$ for almost every $(x, t) \in u^{-1}(E)$. Let $X$ and $Y$ be two nonempty sets. A multifunction $F$ from $X$ into $Y$ (in symbols, $F$ : $\left.X \rightarrow 2^{Y}\right)$ is a function from $X$ into the family of all subsets of $Y$. The graph of $F$ is the set $\{(x, y) \in X \times Y: y \in F(x)\}$. If $W \subseteq Y$ we put $F^{-}(W)=\{x \in X: F(x) \cap W \neq \emptyset\}$. 
When $X$ and $Y$ are two topological spaces we say that $F$ is upper semicontinuous if, for every closed subset $W$ of $Y$, the set $F^{-}(W)$ is closed in $X$. If $(X, \mathcal{F})$ is a measurable space and $Y$ is a topological space, the multifunction $F$ is called measurable provided $F^{-}(W) \in \mathcal{F}$ for every open subset $W$ of $Y$.

In the sequel, we shall apply the following propositions, which are particular cases of classical results on multifunctions (see, for instance, $[8,12]$ ).

Proposition 2.2. Let $X$ be a topological space, let $\varphi: X \longrightarrow \mathbf{R}$ be lower semicontinuous, and let $\psi: X \longrightarrow \mathbf{R}$ be upper semicontinuous. Suppose $\varphi \leqslant \psi$ in $X$. Then the multifunction $F: x \mapsto[\varphi(x), \psi(x)], x \in X$, is upper semicontinuous and the function $x \mapsto d(0, F(x)), x \in X$, is lower semicontinuous.

Propos ITION 2.3. Let $X$ and $Y$ be two topological spaces, let $Y$ be regular, and let $F: X \longrightarrow 2^{Y}$ be an upper semicontinuous multifunction with nonempty and closed values. Then $F$ has a closed graph.

We shall also employ the result below, which is a very special case of [3, Theorem 3.1]. As usual $M\left(Q_{T}\right)$ denotes the family of all (equivalence classes) of measurable functions $w: Q_{T} \longrightarrow \mathbf{R}$.

LEMMA 2.4. Let $U$ be a nonempty set, let

$$
\Phi: U \longrightarrow M\left(Q_{T}\right) \text { and } \Psi: U \longrightarrow L^{P}\left(Q_{T}\right)
$$

be two operators. Finally, let $F: Q_{T} \longrightarrow 2^{\mathrm{R}}$ be a multifunction with nonempty, convex, closed values. Assume that:

(2.4.1) $\Psi$ is bijective and, whenever $v_{h} \rightarrow v$ in $L^{p}\left(Q_{T}\right)$, there exists a subsequence of $\left\{\Phi\left(\Psi^{-1}\left(v_{h}\right)\right)\right\}$ which converges to $\Phi\left(\Psi^{-1}(v)\right)$ at almost all points of $Q_{T}$.

(2.4.2) The set $\{z \in \mathbf{R} \mid F(\cdot, \cdot, z)$ is measurable $\}$ is dense in $\mathbf{R}$.

(2.4.3) $F(x, t, \cdot)$ has a closed graph for almost every $(x, t) \in Q_{T}$.

(2.4.4) There exists a function $m \in L^{p}\left(Q_{T}\right)$ such that

$$
F(x, t, z) \subseteq[-m(x, t), m(x, t)]
$$

for almost all $(x, t) \in Q_{T}$ and each $z \in \mathbf{R}$.

Then the problem $u \in U, \Psi(u)(x, t) \in F(x, t, \Phi(u)(x, t))$ almost everywhere in $Q_{T}$ possesses at least one solution.

\section{REsults}

3.1. The Case of Carathéodory Nonlinearities. In the next theorem we deal with the case when $f$ is a Caratheodory function. Although such a situation has by now been very widely investigated, to the best of our knowledge the result below is new. In particular, we emphasise that, due to the approach adopted, no special growth condition for $f(x, t, \cdot)$ is required. 
THEOREM 3.1. Let $f$ be a real valued function defined on $Q_{T} \times \mathbf{R}$. Assume that:

(3.1.1) For almost every $(x, t) \in Q_{T}$ the function $z \mapsto f(x, t, z)$ is continuous.

(3.1.2) For every $z \in \mathbf{R}$ the function $(x, t) \mapsto f(x, t, z)$ is measurable.

(3.1.3) There exist $\alpha \in L^{p}\left(Q_{T}\right), \beta \in L^{\left(p p^{*}\right) /\left(p^{*}-\gamma p\right)}\left(Q_{T}\right)$, where

$$
\begin{gathered}
p^{\star} \in\left(p, \frac{(n+1) p}{n+1-p}\right) \\
\text { if } p<n+1, p^{\star} \in(p,+\infty) \text { if } p \geqslant n+1,
\end{gathered}
$$

such that

$$
|f(x, t, z)| \leqslant \alpha(x, t)+\beta(x, t)|z|^{\gamma}
$$

for almost all $(x, t) \in Q_{T}$ and every $z \in \mathbf{R}$.

Moreover, if $\|\beta\|_{\left(p p^{*}\right) /\left(p^{*}-\gamma p\right)}>0$ and $\gamma=1$ then $\|\beta\|_{\left(p p^{*}\right) /\left(p^{*}-\gamma p\right)} \cdot c_{1}\left\|\mathcal{L}^{-1}\right\|<1$, while if $\gamma>1$ then

$$
\|\alpha\|_{p} \leqslant \frac{\gamma-1}{\gamma}\left(\frac{1}{\gamma\|\beta\|_{\left(p p^{*}\right) /\left(p^{*}-\gamma p\right)} \cdot c_{1}^{\gamma}\left\|\mathcal{L}^{-1}\right\|^{\gamma}}\right)^{1 /(\gamma-1)} .
$$

Then problem $(P)$ has a solution $u \in W\left(Q_{T}\right)$.

Proof: Define, for every $v \in L^{p}\left(Q_{T}\right)$,

$$
T(v)(x, t)=f\left(x, t, \mathcal{L}^{-1}(v)(x, t)\right),(x, t) \in Q_{T}
$$

Assumptions (3.1.1) and (3.1.2) guarantee that the function $T$ is measurable.

Now write, for any $r>0, K_{r}=\left\{v \in L^{p}\left(Q_{T}\right) \mid\|v\|_{p} \leqslant r\right\}$. Clearly $K_{r}$ is a nonempty, weakly compact, convex subset of $L^{p}\left(Q_{T}\right)$. We show that there exists $r>0$ such that $T\left(K_{r}\right) \subseteq K_{r}$. To this end set

$$
a=\|\alpha\|_{p}, b=\|\beta\|_{\left(p p^{*}\right) /\left(p^{*}-\gamma p\right),} \text { and } c=c_{1}\left\|\mathcal{L}^{-1}\right\|
$$

If $v \in K_{r}$ then

$$
|T(v)(x, t)| \leqslant \alpha(x, t)+\beta(x, t) \cdot\left|\mathcal{L}^{-1}(v)(x, t)\right|^{\gamma}
$$

and using Hölder's inequality yields

$$
\begin{aligned}
\|T(v)\|_{p} & \leqslant\|\alpha\|_{p}+\left\|\beta \cdot\left|\mathcal{L}^{-1}(v)\right|^{\gamma}\right\|_{p} \leqslant a+b\left\|\left|\mathcal{L}^{-1}(v)\right|^{\gamma}\right\|_{p^{*} / \gamma} \\
& \leqslant a+b\left\|\mathcal{L}^{-1}(v)\right\|_{p^{*}}^{\gamma} \leqslant a+b c_{1}^{\gamma}\left\|\mathcal{L}^{-1}(v)\right\|_{2,1, p}^{\gamma} \\
& \leqslant a+b c_{1}^{\gamma}\left\|\mathcal{L}^{-1}\right\|^{\gamma}\|v\|_{p}^{\gamma} \leqslant a+b c^{\gamma} r^{\gamma} .
\end{aligned}
$$

Thus, when $\gamma<1$ the conclusion follows from

$$
\lim _{r \rightarrow+\infty}\left(a+b c^{\gamma} r^{\gamma}-r\right)=-\infty
$$


whereas when $\gamma=1$ we put $r=a /(1-b c)$ and we obtain

$$
\|T(v)\|_{p} \leqslant a+b c \cdot \frac{a}{1-b c}=r .
$$

If $\gamma>1$, choose $r=\left(1 / \gamma b c^{\gamma}\right)^{1 /(\gamma-1)}$. So

$$
\begin{gathered}
\|T(v)\|_{p} \leqslant a+b c^{\gamma} r^{\gamma} \leqslant \frac{\gamma-1}{\gamma}\left(\frac{1}{\gamma b c^{\gamma}}\right)^{1 /(\gamma-1)}+b c^{\gamma}\left(\frac{1}{\gamma b c^{\gamma}}\right)^{1 /(\gamma-1)} \\
=\left(\frac{1}{\gamma b c^{\gamma}}\right)^{1 /(\gamma-1)}\left[\frac{\gamma-1}{\gamma}+\frac{b c^{\gamma}}{\gamma b c^{\gamma}}\right]=r .
\end{gathered}
$$

Hence, in either case, $T\left(K_{r}\right) \subseteq K_{r}$.

Next we prove that $T_{\mid K_{r}}$ is a weakly sequentially continuous operator. To this end, pick a sequence $\left\{v_{h}\right\}_{h \in N}$ in $K_{r}$ weakly converging to $v \in K_{r}$. Due to the weak continuity of $\mathcal{L}^{-1}$ we get $\lim _{h \rightarrow+\infty} \mathcal{L}^{-1}\left(v_{h}\right)=\mathcal{L}^{-1}(v)$ weakly in $W\left(Q_{T}\right)$ and, by the Rellich-Kondrachov theorem, the set $\bigcup_{h \in N}\left\{\mathcal{L}^{-1}\left(v_{h}\right)\right\}$ is relatively compact in $L^{p}\left(Q_{T}\right)$. Thus, there exists a subsequence converging to $\mathcal{L}^{-1}(v)$ almost everywhere. Using (3.1.1) and taking a subsequence if necessary, it follows that

$$
\lim _{h \rightarrow+\infty} T\left(v_{h}\right)(x, t)=T(v)(x, t)
$$

almost everywhere in $Q_{T}$. Now, by the Arino-Gautier-Penot theorem [2, Theorem 1] there exists $v \in K_{r}$ such that $T(v)=v$. The function $u=\mathcal{L}^{-1}(v)$ lies in $W\left(Q_{T}\right)$ and

$$
\mathcal{L}(u)(x, t)=v(x, t)=T(v)(x, t)=f(x, t, u(x, t)) \text { almost everywhere in } Q_{T} .
$$

3.2. The Case of discontinuous (WITh Respect to $u$ ) nonlinearities. Given a real function $f$ defined on $Q_{T} \times \mathbf{R}$, we put, for every $(x, t, z) \in Q_{T} \times \mathbf{R}$

$$
\underline{f}(x, t, z)=\liminf _{w \rightarrow z} f(x, t, w), \bar{f}(x, t, z)=\limsup _{w \rightarrow z} f(x, t, w)
$$

and explicitly observe that the function $z \mapsto \underline{f}(x, t, z)$ is lower semicontinuous, while the function $z \mapsto \bar{f}(x, t, z)$ is upper semicontinuous. Moreover, for every $(x, t, z) \in Q_{T} \times \mathbf{R}$, we define

$$
F(x, t, z)=[\underline{f}(x, t, z), \bar{f}(x, t, z)]
$$

LEMmA 3.2. Let $f$ be a real valued function defined on $Q_{T} \times \mathbf{R}$. Assume that:

(3.2.1) There exists a subset $A \subseteq \mathbf{R}$, with $|A|=0$, such that the function $(x, t) \mapsto$ $f(x, t, z)$ is measurable for every $z \in \mathrm{R} \backslash A$. 
(3.2.2) There is $\alpha \in L^{p}\left(Q_{T}\right)$ such that

$$
|f(x, t, z)| \leqslant \alpha(x, t)
$$

for almost every $(x, t) \in Q_{T}$, for every $z \in \mathbf{R}$.

Then there exists $u \in W\left(Q_{T}\right)$ satisfying

$$
\mathcal{L}(u)(x, t) \in F(x, t, u(x, t))
$$

almost everywhere in $Q_{T}$.

Proof: We prove that all the assumptions of Lemma 2.4 are satisfied. To this end choose $U=W\left(Q_{T}\right), \Phi(u)=u, \forall u \in U, \Psi=\mathcal{L}$. Let $v \in L^{p}\left(Q_{T}\right)$ and let $\left\{v_{h}\right\}_{h \in \mathbb{N}}$ be a sequence in $L^{P}\left(Q_{T}\right)$ weakly converging to $v$. Then $\mathcal{L}^{-1}\left(v_{h}\right) \rightarrow \mathcal{L}^{-1}(v)$ in $W_{p}^{2,1}\left(Q_{T}\right)$. Bearing in mind the Rellich-Kondrachov theorem, there exists a subsequence of $\left\{\mathcal{L}^{-1}\left(v_{h}\right)\right\}_{h \in \mathbf{N}}$ which converges almost everywhere in $Q_{T}$ to $\mathcal{L}^{-1}(v)$. Thus (2.4.1) holds. By hypothesis (3.2.1) we obtain

$$
\{z \in \mathbf{R} \mid F(\cdot, \cdot, z) \text { is measurable }\} \supseteq \mathbf{R} \backslash A,
$$

so (2.4.2) holds true too. Thanks to Propositions 2.2 and $2.3,(2.4 .3)$ is obviously satisfied. Finally, condition (2.4.4) comes immediately from the inequality $|f(x, t, z)| \leqslant \alpha(x, t)$, $(x, t) \in Q_{T}, z \in \mathbf{R}$. Therefore, Lemma 2.4 yields $u \in W\left(Q_{T}\right)$ such that

$$
\mathcal{L}(u)(x, t) \in F(x, t, u(x, t)) \text { for almost every }(x, t) \in Q_{T} .
$$

REMARK 3.1. It is worthwhile to note that the classical Stefan problem can be reduced to a problem of the type studied in Lemma 3.2; see [6, p. 4]. Moreover, [6, Theorem 4.3] gives a solution to such a problem only when $p>n$ while Lemma 3.2 can be applied for any $p>1$.

REMARK 3.2. The discontinuous nonlinearity treated in $[4,16]$ is of the form $f(u)+$ $h(x, t)$, where $f \in L_{l o c}^{\infty}(\mathbf{R})$, while the boundedness condition required is

$$
|f(z)| \leqslant \beta(x, t), z \in[\underline{u}(x, t)-\alpha, \bar{u}(x, t)+\alpha],
$$

$\underline{u}$ and $\bar{u}$ being a lower solution and an upper solution, respectively, to the problem considered there. Although this inequality is formally more general than $b$ ) of Lemma 3.2, the results of $[4, \mathbf{1 6}]$ exhibit several further restrictions that prevent us treating the general case.

We now come to problem $(P)$ with $f$ possibly highly discontinuous in $u$.

THEOREM 3.3. Let $f$ be a real-valued function defined on $Q_{T} \times \mathbf{R}$ having the following properties: 
(3.3.1) There exists a set $Q_{0} \subseteq Q_{T}$ with $\left|Q_{0}\right|=0$ such that the set

$$
D_{f}=\bigcup_{(x, t) \in Q_{T} \backslash Q_{0}}\{z \in \mathbf{R} \mid f(x, t, \cdot) \text { is discontinuous at } z\}
$$

has measure zero.

(3.3.2) The function $(x, t) \mapsto f(x, t, z)$ is measurable for every $z \in \mathbf{R} \backslash D_{f}$.

(3.3.3) There exists a function $\alpha \in L^{p}\left(Q_{T}\right)$ such that

$$
|f(x, t, z)| \leqslant \alpha(x, t)
$$

for almost every $(x, t) \in Q_{T}$, for every $z \in \mathbf{R}$.

(3.3.4) There exists a set $Q_{1} \subseteq Q_{T}$ of measure zero such that for every $(x, t) \in$ $Q_{T} \backslash Q_{1}$ and every $z \in D_{f}$, the condition $0 \in F(x, t, z)$ implies $f(x, t, z)=0$. Then problem $(P)$, with $\mathcal{L}$ replaced by $\tilde{\mathcal{L}}=\mathcal{L}-a(x, t) u$, has a solution $u \in W\left(Q_{T}\right)$.

PRoOF: Thanks to Lemma 3.2, applied with $A=D_{f}$, there exists a function $u \in$ $W\left(Q_{T}\right)$ such that $\tilde{\mathcal{L}}(u)(x, t) \in F(x, t, u(x, t))$ for almost every $(x, t) \in Q_{T}$. Let $Q_{2} \subseteq Q_{T}$ be such that $\left|Q_{2}\right|=0$ and $\tilde{\mathcal{L}}(u)(x, t) \in F(x, t, u(x, t))$ for every $(x, t) \in Q_{T} \backslash Q_{2}$ and let

$$
Q_{f}=\left\{(x, t) \in Q_{T} \backslash Q_{0} \mid u(x, t) \in D_{f}\right\} .
$$

Due to Proposition 2.1 there exists a set $Q_{3} \subseteq Q_{f}$ such that $\left|Q_{3}\right|=0$ and $\widetilde{\mathcal{L}}(u)(x, t)=0$ for every $(x, t) \in Q_{f} \backslash Q_{3}$. Now, define $Q^{*}=\bigcup_{i=0}^{3} Q_{i}$; obviously, one has $\left|Q^{*}\right|=0$. We prove that

$$
\tilde{\mathcal{L}}(u)(x, t)=f(x, t, u(x, t)) \text { for every }(x, t) \in Q_{T} \backslash Q^{*} .
$$

Pick $(x, t) \in Q_{T} \backslash Q^{*}$. If $(x, t) \notin Q_{f}$ then $u(x, t) \notin D_{f}$ and, consequently, $F(x, t, u(x, t))=$ $\{f(x, t, u(x, t))\}$. If $(x, t) \in Q_{f}$ then $(x, t) \notin Q_{3}, \tilde{\mathcal{L}}(u)(x, t)=0$ and, by virtue of (3.3.4), $f(x, t, u(x, t))=0$. So, once again, $\widetilde{\mathcal{L}}(u)(x, t)=f(x, t, u(x, t))$.

REMARK 3.3. We observe that assumptions (3.3.1), (3.3.2), and (3.3.3) immediately lead to an existence result for the following problem:

$$
\begin{gathered}
\frac{\partial u}{\partial t}= \begin{cases}\Delta u+f(x, t, u) & \text { almost everywhere in }\left\{(x, t) \in Q_{T}: u(x, t) \notin D_{f}\right\} \\
0 & \text { almost everywhere in }\left\{(x, t) \in Q_{T}: u(x, t) \in D_{f}\right\}\end{cases} \\
0 \in[f(x, t, u), \bar{f}(x, t, u)] \text { almost everywhere in }\left\{(x, t) \in Q_{T}: u(x, t) \in D_{f}\right\} .
\end{gathered}
$$

A similar problem is treated in [9, Theorem 2.13], but to a more general growth condition on the nonlinear term, which depends only on $(x, z)$, there corresponds a stronger restriction on $p$. Namely, in [9] one has:

$$
|f(x, z)| \leqslant a(x)+b|z|^{p}, \text { with } a \in L^{2}(\Omega), p \in\left[1, \frac{n}{n-2}\right) \cap\left[1,1+\frac{4}{n}\right) .
$$


REMARK 3.4. We explicitly point out that the conditions imposed on $f$ in [5] prevent us considering functions like $\alpha(x, t) g(z)$, with $g$ nonmonotone. Hence, the set of discontinuities of $g$ is at most countable. On the contrary, Theorem 3.3 can be applied also when the nonlinearities involved have an uncountable set of discontinuity points, as the next example shows.

Example 3.1. Let $C$ be the Cantor 'middle thirds' set. It is known that $C$ is closed, uncountable, and of measure zero. So $\mathbf{R} \backslash C$ is nonempty, open, and has at most countably many connected components $A_{h}, h=1,2, \ldots$ Pick $y^{\star}>0$ and a bounded sequence $\left\{y_{h}\right\}_{h \in \mathbb{N}}$ such that

$$
\inf _{h \in \mathbb{N}} y_{h}>0, y^{\star} \notin\left[\inf _{h \in \mathbf{N}} y_{h}, \sup _{h \in \mathbf{N}} y_{h}\right]
$$

and define

$$
g(z)= \begin{cases}y_{h} & \text { if } z \in A_{h} \\ y^{\star} & \text { if } z \in C .\end{cases}
$$

Now, set $f(x, t, z)=\alpha(x, t) g(z)$, where $\alpha \in L^{p}\left(Q_{T}\right)$, and $\alpha \geqslant 0$. The function $f$ satisfies all the assumptions of Theorem 3.3 , because $D_{f}=C$,

$$
|f(x, t, z)| \leqslant \sup _{h \in \mathbb{N}} y_{h} \cdot \alpha(x, t),
$$

and $0 \in[f(x, t, z), \bar{f}(x, t, z)]$ if and only if $\alpha(x, t)=0$, that is $f(x, t, z)=0$.

REMARK 3.5. An easy computation ensures that if there exists an open subset $A$ of $\mathrm{R}$ such that $D_{f} \subseteq A$ and

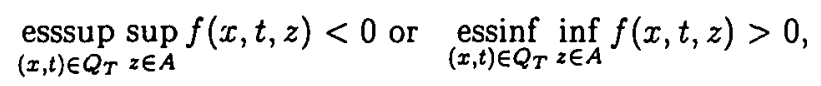

then hypothesis (3.3.4) is satisfied.

\section{REFERENCES}

[1] R.A. Adams, Sobolev spaces, Pure and Applied Mathematics (Academic Press, New York, London, 1975).

[2] O. Arino, S. Gautier and J.P. Penot, 'A fixed point theorem for sequentially continuous mappings with applications to ordinary differential equations', Funkcial. Ekvac. 27 (1984), 273-279.

[3] D. Averna and S.A. Marano, 'Existence theorems for inclusions of the type $\Psi(u)(t) \in F(t, \Phi(u)(t))$ ', Appl. Anal. 72 (1999), 449-458.

[4] S. Carl, 'Enclosure of solutions for quasilinear dynamic hemivariational inequalities', Nonlinear World 3 (1996), 281-298. 
[5] S. Carl and S. Heikkilä, 'Extremal solutions of quasilinear parabolic boundary value problems with discontinuous nonlinearities', Dynamic Systems Appl. 3 (1994), 251-258.

[6] K.C. Chang, 'Free boundary problems and the set-valued mappings', J. Differential Equations 49 (1983), 1-28.

[7] E. De Giorgi, G. Buttazzo and G. Dal Maso, 'On the lower semicontinuity of certain integral functionals', Atti Accad. Naz. Lincei Cl. Sci. Fis. Mat. Natur. Rend. Lincei (8) Mat. Appl. 74 (1983), 274-282.

[8] K. Deimling, Multivalued differential equations, de Gruyter Series Nonlin. Anal. Appl. 1 (Walter de Gruyter, Berlin, 1990).

[9] M. Frigon and C. Saccon, 'Heat equations with discontinuous nonlinearities on convex and non convex constraints', Nonlinear Anal. 17 (1991), 923-946.

[10] D. Gilbarg and N.S. Trudinger, Elliptic partial differential equations of second order, Second edition (Springer-Verlag, Berlin, Heidelberg, New York, 1983).

[11] S. Heikkilä and V. Lakshmikantham, Monotone iterative techniques for discontinuous nonlinear differential equations (Marcel Dekker, New York, 1994).

[12] E. Klein and A.C. Thompson, Theory of correspondences (Wiley, New York, 1984).

[13] O.A. Ladyženskaja, V.A. Solonnikov and N.N. Ural'ceva, Linear and quasilinear equations of parabolic type, Transl. Math. Monographs 23 (Amer. Math. Soc., Providence, R.I., 1968).

[14] S.A. Marano, 'Elliptic boundary-value problems with discontinuous nonlinearities', Set-Valued Anal. 3 (1995), 167-180.

[15] S.A. Marano, 'Existence results for the equation $-\Delta u=f(x, u)$ in $R^{n}$, $Z$. Anal. Anwendungen 19 (2000) (to appear).

[16] N.S. Papageorgiou, 'On the existence of solutions for nonlinear parabolic problems with nonmonotone discontinuities', J. Math. Anal. Appl. 205 (1997), 434-453.

Dipartimento di Matematica

Università di Messina

contrada Papardo

salita Sperone n.31

98166 Sant' Agata (ME)

Italy

e-mail: barletta@dipmat.unime.it
Dipartimento di Informatica

Matematica, Elettronica e Trasporti

Università di Reggio Calabria

Via Graziella (Feo di Vito)

89100 Reggio Calabria

Italy

e-mail: barletta@ing.unirc.it 